\title{
Reducing Burden, Ensuring Competence, Improving Quality, and Enhancing Professionalism: How Can ABFM Contribute to All?
}

Robert Phillips, MD, MSPH

For many physicians, maintaining certification is but 1 of a number of nonclinical activities that require additional time and energy. The American Board of Family Medicine (ABFM) is working to balance the importance of providing a mechanism for family physicians to demonstrate their ongoing competency and improvement with the additional burden and expense that federal efforts to move toward value-based payment will add to physicians' work. For this reason, the ABFM is investing heavily to align continuous certification with valuebased payment requirements in a manner that both reduces burden and bends value-based payments to primary care's value. A description of these multifaceted efforts is described below.

\section{PRIME Registry}

For family physicians who have difficulty retrieving data for quality reporting from their electronic health records (EHRs), ABFM is providing tools to turn EHR data into information. PRIME dashboards inform patient and population quality, and its measures populate Maintenance of Certification portfolios to tailor quality improvement choices to the most meaningful activities. These data are reported at the physician's discretion to meet valuebased payment reporting and other needs. Through the PRIME registry, we will merge data from EHRs with measure submissions to produce a more inclusive registry. More information is available at www.theabfm.org/primeregistry/.

\section{TRADEMaRQ}

TRADEMaRQ (Trial of Aggregate Data Exchange for MOC and Raising Quality) refers to a federally funded study that seeks to answer 3 questions: (1)

Conflict of interest: The author is affiliated with the ABFM.
Can family physicians who are in systems that measure quality send whole-panel quality measures to the ABFM? (2) Can those data be used to better address quality improvement needs at the practice level? (3) Will this process result in improved quality? Participation in TRADEMaRQ is 1 path into the PRIME Registry.

\section{PRIME Support and Alignment Network}

The ABFM partnered with the American Academy of Family Physicians to support the large federal effort to help practices succeed with value-based payments. The ABFM saw this as a way to secure important resources for helping family medicine practices and thereby to assist with reducing the burden and planning required for family physicians to accomplish practice improvement activities. ABFM is giving Part IV credit and making the PRIME Registry free for 6000 board certified family physicians who join a practice transformation network.

\section{Measure Development}

The PRIME Registry is a federally certified qualified clinical data registry, which means it can propose to the Centers for Medicare \& Medicaid Services better primary care measures for payment. ABFM's research collaborations with the Robert Graham Center prepare and allow us to produce better measures for value-based payment. Having the data, the capacity to analyze them, and the ability to develop/test measures will help us recommend pay-for-performance measures that family physicians believe are important. ABFM wants meaningful quality improvement to count for multiple things, so we hope, over time, to move practice improvement activities (Part IV) into the PRIME Registry with the goal that when a family physician completes a Performance in Practice 
module, they also complete a clinical practice improvement activity ( 1 of the new value-based payment measures).

\section{Population Health}

The ABFM is working to integrate social determinants of health data with clinical data in the PRIME Registry. The goal is to help prepare family medicine practices for understanding the impact of social determinants on the individual patients and populations they serve so that interventions to improve care can be planned with these in mind. Through this effort, community-level quality improvement efforts that count for maintaining certification and value-based payments will be developed. This also helps with preparation for the payment adjustments based on social determinants expected in 2017.

\section{Evolution of Continuous Certification}

The ABFM uses research and feedback from its diplomates to continuously improve continuous certification. More than $80 \%$ of diplomates told us that the knowledge self-assessment portion of Part II was valuable for updating their knowledge, but only $60 \%$ said the same for the clinical skills portion. In response, the ABFM is separating these 2 components and making them independent options. We are working on another Part II solution that allows diplomates to receive questions on their mobile devices and answer fewer knowledge self-assessment questions, albeit more often. Other boards have found that their diplomates favor this method, and educational research supports it for improving learning. Our internal research will also lead to improvements in the certification examination, which will be announced later this year.

Certifying boards can, and should, play a critical role in reducing burden and burnout by helping physicians demonstrate ongoing competency and respond to requirements for improving care quality. The ABFM is committed to helping its diplomates through work at the practice and policy levels on their behalf, and we welcome their partnership in this process.

Acknowledgement: TRADEMaRQ was supported by grant number R21HS022583 from the Agency for Healthcare Research and Quality. The content is solely the responsibility of the authors and does not necessarily represent the official views of the Agency for Healthcare Research and Quality. 